\title{
LA CREACIÓN DE ORGANIZACIONES DE ALTO DESEMPEÑO (OAD) BASADAS EN LA GESTIÓN ECONÓMICA DEL CONOCIMIENTO
}

CREATING HIGH PERFORMANCE ORGANIZATIONS (HPO) BASED ON ECONOMIC KNOWLEDGE MANAGEMENT

\section{CECILIA VÉlEZ B.', CARLOS TRIVIÑo I. ${ }^{2}$, EVELYN GARCÍA M. ${ }^{3}$, JULIO JÁCOME T. ${ }^{4}$}

\footnotetext{
Universidad Católica de Santiago de Guayaquil. cecilia.velez@cu.ucsg.edu.ec

Universidad Católica de Santiago de Guayaquil. carlos.trivino@cu.ucsg.edu.ec

3 Universidad de Guayaquil.evelyn.garciam@ug.edu.ec

4 Asesor Empresarial.jacome.julio@cu.ucsg.edu.ec
}

\section{RESUMEN}

La presente investigación trata temas de vital importancia para el crecimiento económico de los países, los elementos básicos de la relación universidad-empresa-sociedad en la búsqueda de la utilización efectiva del conocimiento y la innovación tecnológica como factor de progreso en los países. El objetivo de este artículo es esbozar las perspectivas de la economía ecuatoriana en función del conocimiento, alineada a la actualización del modelo económico a través de una correcta gestión organizacional sobre la base del potencial científico técnico alcanzado. Dejando fuera recetas neoliberales que separan por completo el mundo físico del razonamiento económico y abocado a la creación de las condiciones para la realización de la personalidad humana en su interacción con el Medio Ambiente favoreciendo el desarrollo sostenible del país.

PALABRAS CLAVE: economía del conocimiento, gestión organizacional, alto desempeño, universidad.
ABSTRACT

This research addresses issues of vital importance to the economic growth of countries, the basic elements of the university-business-society in the search for effective use of knowledge and technological innovation as a factor of progress of the countries. The objective of this research is to outline the prospects of the Ecuadorian economy in terms of knowledge, aligned to the economic model updating through proper organizational management based on scientific and technical potential reached. Leaving out neoliberal recipes that completely sever the physical world of economic reasoning and devoted to the creation of conditions for the realization of human personality in its interaction with the environment by promoting sustainable development in the country.

KEYWORDS: knowledge economy, organizational management, high performance, university. 
INTRODUCCIÓN

La tendencia actual en la evolución de las economías, depende en gran medida de la utilización efectiva del conocimiento y la innovación como factor de progreso para los países. Al analizar la velocidad de generación de conocimientos y tecnologías en la actualidad, se observa que sobrepasa la velocidad de difusión de ese conocimiento nuevo generado. Este enfoque circunstancial obliga a revalorizar el rol que desempeña el conocimiento en el crecimiento económico y asociado a él, la gestión del talento humano y su capacidad de generación en las nuevas formas de organización.

Muchos han sido los autores que han escrito acerca de las economías basadas en el conocimiento y sus profundas implicaciones para los factores de producción, la organización de la producción y sus efectos sobre el empleo y las calificaciones requeridas, y puede llegar a demandar nuevas orientaciones de política industrial, cómo lo explica la Organización para la Cooperación y el Desarrollo Económicos, OCDE (1996), además de reducir las necesidades de materias primas, fuerza de trabajo, tiempo, espacio y capital, disminuyendo los costos y centrando al conocimiento en la médula de las economías de avanzada.

$\mathrm{Al}$ analizar los antecedentes (Parques y Distritos Tecnológicos, Sistemas Nacionales de Innovación, etc.) existentes entre la investigación científica y la economía en diferentes países por la naturaleza del contexto en que se desarrollan es limitado, pues lo que se presencia en la función del conocimiento de la economía no es un cambio incremental sino una discontinuidad. Las discontinuidades no se dejan explorar fácilmente con extrapolaciones de tendencias pasadas. Por ello, la correcta utilización del conocimiento como recurso productivo en relación a los tradicionales, es proceso de análisis en el contexto actual.

La expresión "economía basada en los conocimientos" capta una diferencia cualitativa en la organización y conducta de la vida económica moderna. Los que utilizan la expresión sostienen que los determinantes del éxito de las empresas y del conjunto de la economía de un país, dependen cada vez más de su efectividad para generar y utilizar conocimientos (Lundvall 1992). A pesar de que el conocimiento científico y tecnológico tiene una importancia clave, el conocimiento acerca de cómo organizar y gestionar las actividades económicas, especialmente aquellas que comprenden la aplicación de nuevas perspectivas científicas y tecnológicas, también es un determinante crucial del rendimiento económico. Este progreso organizacional y de gestión está cobrando importancia a medida que aumenta el contenido científico y tecnológico de la actividad económica.

La economía del conocimiento por su influencia no es un hecho consolidado hasta la fecha y se agrava con las desigualdades en el desarrollo de la

Humanidad, por lo que todo país tiene la obligación de guiar su proceso de crecimiento, es decir contar con un modelo adaptado a sus condiciones objetivas.

DESARROLLO

Para llevar a cabo el objetivo de la investigación de la necesidad de analizar las perspectivas de la economía ecuatoriana basada en el conocimiento y así crear la base organizativa para el diseño de empresas de alta tecnologías. Para ello se estudiaron las principales teorías del crecimiento económico mediante la observación científica, análisis de contenido y síntesis de la problemática del crecimiento económico que va más allá de una visión global del mismo, intentando explorar interioridades pasadas y presentes, todo esto como primer eslabón de la cadena. Para luego enfocar cuál es el papel de la universidad en el crecimiento como segundo eslabón en la concepción de los nexos internos y externos del mismo. Luego culminar con un somero análisis de las bases organizativas de estas organizaciones y algunas soluciones que todavía se quedan a nivel macro para posteriores trabajos.

\section{MODELOS DE CRECIMIENTO ECONÓMICO} EL CRECIMIENTO ECONÓMICO DE UN PAÍS

Se define como el aumento de la renta o valor de bienes y servicios finales producidos por una economía (generalmente un país o una región) en un determinado período, es decir, la reproducción a escala ampliada del valor de la producción con respecto a un período. Varios neoclásicos del siglo pasado como Keynes (1936), Solow (1956), y Swan (1956) explican que los factores productivos básicos determinantes del crecimiento económico son la tierra, el capital y el trabajo. Todo lo anterior basado en lo demostrado por clásicos de la economía como David 
Ricardo (2003) y Smith (1983); en condiciones de una economía con equilibrio estacionario. ${ }^{1}$

Solow (1956) en su modelo cuantitativo estableció la función de producción (Yt = F (Kt, Lt, A)2 ${ }^{2}$ como eje esencial del crecimiento económico de los Estados Unidos de América (EUA), criticando el modelo keynesiano y explicando que el incremento de la productividad y la renta per cápita era consecuencia del progreso tecnológico3, el cual según su teoría crecía de forma exógena y por el aumento de la relación capital/trabajo. Este progreso se denominó como un "residuo" de la función de producción indicando todas aquellas fuerzas distintas del capital físico y el trabajo que influyen sobre el crecimiento. Al tomar como base este modelo la Ley de los rendimientos decrecientes $4{ }^{4}$ tuvo una consecuencia nefasta, pues el crecimiento a largo plazo debido a la acumulación de capital era insostenible. A partir de la década del 80 del pasado siglo, Romer (1986) y Lucas (1988) tuvieron como objetivo esencial la construcción de modelos en los que a diferencia de los modelos neoclásicos, la tasa de crecimiento a largo plazo fuera positiva sin la necesidad de suponer que alguna variable del modelo crecía de forma exógena, eliminando la tendencia de los rendimientos decrecientes del capital, al suponer que el conocimiento era obtenido como un subproducto de la inversión en capital físico. Estas nuevas teorías se denominaron "crecimiento endógeno".

1 Considera que el desempeño a largo plazo se caracteriza por una tasa de crecimiento común para todos los factores.

2 El stock de capital (K), el trabajo (L) y el tiempo (t), parte de los supuestos microeconómicos neoclásicos sobre la producción en los que se produce un bien compuesto $(\mathrm{Y})$ a partir de dos bienes simples intercambiables entre sí como son el capital y el trabajo.

3 Es todo tipo de desplazamiento de la función producción (hacia arriba). Retardos y aceleraciones, mejoras en la calificación de mano de obra y todo tipo de factores aparecerán como "cambio técnico"”. Cambio del proceso de producción o introducción de nuevos productos que permiten obtener un nivel producción mayor o mejor con la misma cesta de factores".

4 Situación en la cual los aumentos en la cantidad de un factor productivo variable se combinan con cantidades fijas de otros factores, y como resultado, el rendimiento marginal, primero, y más tarde el rendimiento medio del factor variable disminuyen a partir de un momento dado.
Según un estudio realizado por Moreno (2013), y en común acuerdo con los miembros de la teoría endógena de crecimiento económico, se cree que en el Ecuador es ilógico consentir que el esfuerzo inversor, los procesos de investigación y desarrollo (I+D), el gasto público o la fiscalidad no tengan ningún efecto a largo plazo sobre la tasa de crecimiento; tampoco se cree que el accionar del gobierno no afecte a la economía. Por tal motivo, el modelo supone que el accionar estatal puede afectar positiva o negativamente al crecimiento de largo plazo de la economía ecuatoriana, a través, de la implementación de políticas como la monetaria, fiscal y estructural.

Ahora, un elemento importante del crecimiento en el modelo de Romer está determinado por la acumulación de conocimientos en los individuos, lo cual se constituye en el "motor del crecimiento" de las economías de los países, pero a la vez explica que las multinacionales obtienen provecho de la situación acumulando para sí, los recursos humanos de los países subdesarrollados y el capital humano disponible, al tiempo que extraen sus capitales en forma de dividendos hacia el exterior, en vez de reinvertirlos en el país de origen. En consonancia con esto el modelo de Lucas plantea que el crecimiento está representado por el capital humano, el mismo, comprende las capacidades que es capaz de adquirir un individuo en el tiempo, lo cual marca una diferencia con el concepto de capital humano que postula la Escuela Neoclásica.

Realizando consideraciones críticas de estos modelos según lo expuesto, significa que las tasas de crecimiento de los países dependen de forma inversa de su ingreso per cápita inicial y que la disparidad en los niveles de ese ingreso entre dos economías será transitoria en la medida en que las mismas presenten idénticas tecnologías, preferencias y tasas de crecimiento de la población, todo esto según Solow y sus partidarios los cuales proponen modelos susceptibles de generar políticas activas mientras que Romer y Lucas buscan explicar las razones del crecimiento sin intervención. Resumiendo se puede concluir que el crecimiento económico de un país está asociado al progreso tecnológico que obtenga a través del aprovechamiento de las capacidades y habilidades de la población.

Pero el orden imperante donde el juego de los mercados tiende a acrecentar las desigualdades entre ricos y pobres y que estas solo pueden ser 
eliminadas mediante políticas industriales y sociales extremadamente activas.

Para ello se requiere de la construcción y expansión de un sector productor de conocimientos, en el cual la educación superior es clave para el desarrollo.

\section{PAPEL DE LA UNIVERSIDAD EN EL CRECIMIENTO} ECONÓMICO

La economía del conocimiento según Lage (2007) "intenta capturar un conjunto de fenómenos que denotan una importancia creciente del conocimiento en el funcionamiento y desarrollo de los sistemas productivos de bienes y servicios". Torrent (2009) fundamenta que la economía del conocimiento se consolida a través de una nueva propiedad técnica: la relación asociada entre las Tecnologías de las Informática y las Comunicaciones (TIC) y el conocimiento. En otras palabras: la aplicación de nuevos conocimientos e informaciones sobre aparatos de generación de conocimiento y del proceso de la información y la comunicación. Actualmente, la aplicación económica del conocimiento se utiliza, en la generación y aplicación del propio conocimiento.

Las universidades, según sus posibilidades y capacidades, deben desempeñar un papel clave en la contribución al desarrollo de las naciones al elevar su aporte económico, mediante la ejecución de productos y servicios obtenidos de sus investigaciones. Además, tienen que ser capaces de lograr altos niveles de ingresos de capital que pueden ser reinvertidos tanto en los propios procesos investigativos, como en su aporte a la institución y la colectividad social.

Por desgracia, en el contexto actual, el conocimiento está ubicado cómo recurso limitante en la competencia económica y relaciones de poder, experimentándose su apropiación privada y acumulación en los países más industrializados del mundo. Lo anterior se evidencia con lo planteado por Vela (2011) donde alega que en el "Informe sobre la educación superior en Estados Unidos", preparado por la comisión creada por la Secretaria del Departamento de Educación de los EUA Margaret Spelling, se pone de manifiesto que el $30 \%$ de los Doctores en Ciencia e Ingeniería que laboran en disimiles de sus instituciones no son naturales de este país.

El papel estratégico de las universidades, como fuente de conocimiento, es representar su capacidad de realizar investigación y desarrollo. En correspondencia a esto, se observa un tránsito de la forma tradicional de investigación y generación de conocimientos a nuevos modelos de integración Universidad-Empresa-Sociedad, representados en Sistemas Nacionales de Innovación, como por ejemplo el de Lundvall (2000), Arocena y Sutz (2003); en el que el conocimiento se inserta en los procesos y productos, creando una nueva línea de resultados que permitan obtener ventajas competitivas mediante alianzas estratégicas del sector empresarial con la "casa de altos estudios" y los centros de investigación asociados. Su desarrollo a través de constantes intercambios, marcan cada vez más la dinámica económica, social y cultural actual.

Como queda claro según (Navarro, Llisterri y Zúñiga, 2010), la inversión en innovación, la adquisición, absorción, modificación y creación de conocimiento tecnológico y no tecnológico, son actividades indispensables para el desarrollo de cualquier economía.

En este sentido, las universidades se comportan como el motor impulsor para generar conocimientos en la actualidad, más que los recursos naturales o el capital financiero. En esta nueva sociedad, en la que los conocimientos se aplican de forma prácticamente inmediata en la producción, el factor determinante y la ventaja competitiva está precisamente en la generación de conocimientos.

Los países subdesarrollados y en vías de desarrollo avizorando la viabilidad a largo plazo de los sistemas económicos tienen la necesidad de construir sus propios sistemas e interrelaciones donde la investigación y la innovación sean el fundamento para su desarrollo sostenible.

En el contexto ecuatoriano, según artículo publicado, en el Banco Interamericano de Desarrollo División de Competitividad e Innovación, por Guaipatin y Schwartz (2014), se evidencia una creciente inversión pública sin precedentes en Ecuador en actividades de innovación y en la creación de una base de talento humano altamente calificado. Este análisis se beneficia de información cuantitativa inédita sobre el tema de emprendimiento, y resalta la presencia de una masa crítica de emprendedores que están innovando y generando oportunidades de crecimiento para el país especialmente en el sector servicios. 


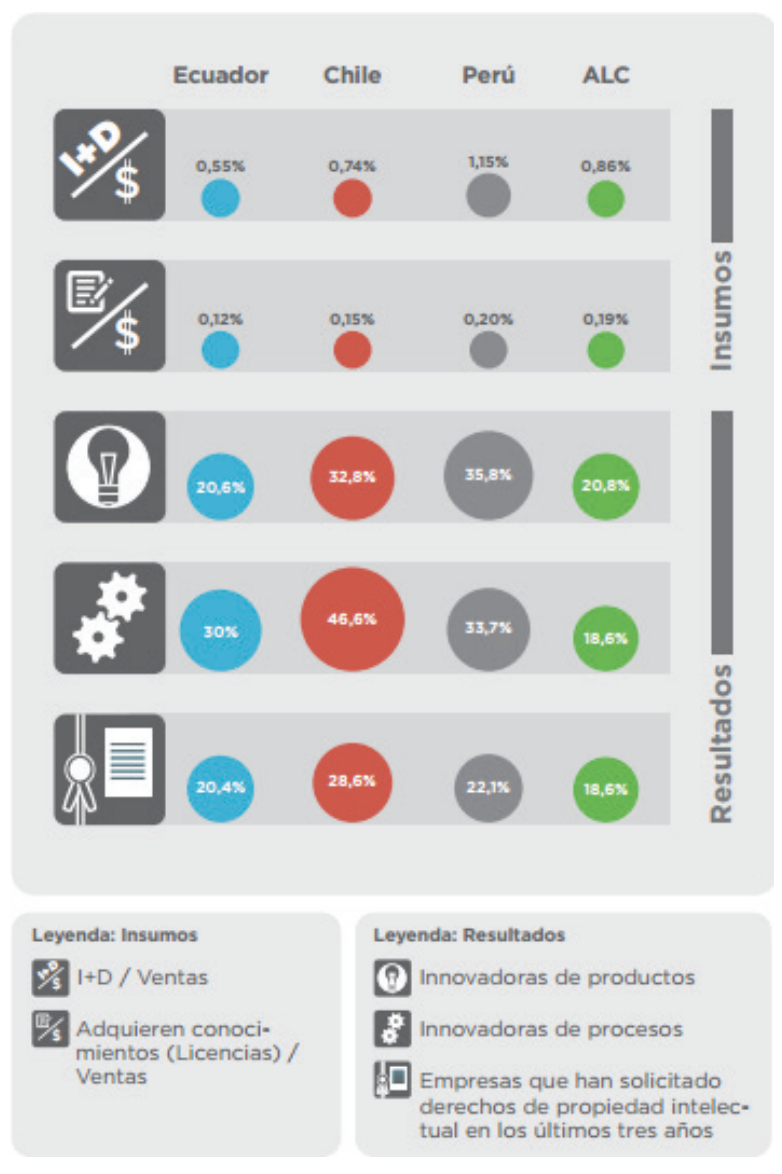

Figura 1. Comparación en innovación en empresas de Ecuador, Chile y Perú. Fuente: Guaipatin y Schwartz (2014).

Sin embargo, la infografía (Figura 1) muestra cómo aún en Ecuador la conectividad de banda ancha y el uso productivo de las TIC son modestos. En comparación con la región, las empresas no tienen un comportamiento muy innovador, lo cual se observa tanto en su nivel de inversión en insumos (por ejemplo, I+D y licencias) como en sus resultados de innovación en productos y procesos, y en la solicitud de patentes.

Sin embargo, a pesar de esto, del año 2012 al 2013, en los datos presentados para Ecuador en el ranking mundial de países mejor calificados en innovación, este pasó de la posición 98 a la 83, es decir, escaló 15 posiciones en un solo año. Esto indica que el Ecuador, se está proyectando para alcanzar una economía basada en la gestión eficaz del conocimiento y la innovación, tratando de dejar atrás ese círculo vicioso actual que se establece entre el déficit comercial, $\mathrm{pa}^{-}$ trón de especialización y patrón de aprendizaje tecnológico.
En la Tabla 1, se puede apreciar parte del Informe de Medición de la Sociedad de la Información del año 2014, Brahima Sanou (2014), desarrollado por la Unión Internacional de Telecomunicaciones (UIT). Uno de los parámetros más importantes, es el Índice de Desarrollo de las TIC (IDI) que mide el progreso de los países en la sociedad de la información. Este índice está compuesto de 11 indicadores que miden el acceso, uso y conocimiento de las TIC. En el subíndice del IDI: Conocimientos, para la Región de las Américas, el Ecuador aparece en el lugar 16 en el 2008 y en el lugar 19 en el 2013.

TABLA 1. FRAGMENTO DEL IDI DE LAS AMÉRICAS REFERENTE AL SUBÍNDICE DEL CONOCIMIENTO.

\begin{tabular}{llll}
\hline ECONOMía & $\begin{array}{l}\text { RANK } \\
\text { REGIONAL }\end{array}$ & $\begin{array}{l}\text { RANK } \\
\text { GLOBAL }\end{array}$ & IDI \\
& $\mathbf{2 0 1 3}$ & $\mathbf{2 0 1 3}$ & \\
\hline United States & 1 & 14 & 8.02 \\
\hline Canada & 2 & 23 & 7.62 \\
\hline Barbados & 3 & 35 & 6.95 \\
\hline Uruguay & 4 & 48 & 6.32 \\
\hline St. Kitts and Nevis & 5 & 54 & 6.01 \\
\hline Costa Rica & 6 & 55 & 5.92 \\
\hline Chile & 7 & 56 & 5.92 \\
\hline Antigua \& Barbuda & 8 & 57 & 5.89 \\
\hline Argentina & 9 & 59 & 5.80 \\
\hline Brasil & 10 & 65 & 5.50 \\
\hline Trinidad \& Tobago & 11 & 67 & 5.29 \\
\hline St. Vicent and the Grenadines & 12 & 72 & 5.17 \\
\hline Grenada & 13 & 76 & 4.96 \\
\hline Colombia & 14 & 77 & 4.95 \\
\hline St. Lucia & 15 & 79 & 4.81 \\
\hline Venezuela & 16 & 80 & 4.81 \\
\hline Panamá & 17 & 82 & 4.75 \\
\hline Dominica & 18 & 83 & 4.72 \\
\hline Ecuador & 19 & 88 & 4.56 \\
\hline
\end{tabular}

Fuente: Informe Mundial de Medición de las TIC por la Unión Internacional de Telecomunicaciones (UIT) 2014.

Finalmente, apoyando a León Guzmán (2014), en Ecuador un crecimiento conducido por el conocimiento y la innovación requiere potenciar la creación de un sistema nacional de innovación, es decir, una red de instituciones del sector público, sector privado y academia 
que interactúen en la producción, difusión y uso de los nuevos conocimientos, tecnologías e innovaciones.

\section{ORGANIZACIONES DE ALTA TECNOLOGÍA. BASES} ORGANIZATIVAS

Los sectores y productos que constituyen la denominada alta tecnología se pueden definir genéricamente como aquellos que dado su grado de complejidad requieren un continuo esfuerzo de investigación e innovación y una sólida base tecnológica. En los llamados "sectores de alta tecnología" en cuyas empresas pueden distinguirse las siguientes características:

- Altos gastos en Investigación-Desarrollo (I+D).

- Oferta de productos y servicios especializados, a veces únicos.

- Competencia por diferenciación de productos, más que por escala y precio.

- Frecuentes negociaciones sobre "activos intangibles" (patentes, marcas, tecnologías, etc.).

- Amplio uso de la protección de patentes.

- Aceptación del riesgo como consustancial a la gestión empresarial.

- Empleo de fuerza de trabajo de alta calificación.

- Los recursos humanos son irremplazables: se tratan como un "activo" y no como un "costo".

Estas dimensiones han demandado de los grupos y centros deinvestigaciones el aprendizajede asuntos en los cuales antes no habían incursionado: estudios de mercado, análisis de costos, evaluación de proyectos, gestión de la calidad, estrategias de comercialización, contratos, propiedad intelectual, licencias, publicidad, entre muchos otros. Ocurre así una transformación en la racionalidad y la cultura de varios de los líderes científicos e investigadores. El trabajo multidisciplinario con economistas, juristas, especialistas en mercadotecnia, que trabajan en la propia Universidad, ha constituido un respaldo importante.

De igual forma se pueden establecer analogías con el sector de las TIC basadas en las características y condiciones que deben demostrar las organizaciones de alta tecnología. En el caso específico de Ecuador se requiere además la inversión en infraestructuras y acceso a las mismas. Contar con equipos de alto desempeño que desarrollen soluciones creativas, yendo más allá de una constante actualización en conocimientos técnicos, sino sobre la base de la capacidad de comprender y anticipar el cambio, mediante la inversión en I+D como señalaran Castro Díaz-Balart y Pérez (2006) al alegar que como mínimo, el 4\% del PIB debe invertirse en la educación, y el 0,64\% de éste en la investigación científica, según se estableció en el Proyecto Milenio de las Naciones Unidas.

El sector de las TIC en el país debe orientar su gestión de forma inmediata para convertirse en una red de organizaciones de alta tecnología. Las universidades ecuatorianas como parte de este sector deben dirigir sus políticas y objetivos para alcanzar un alto grado de aprovechamiento de sus capacidades de formación, desarrolloproducción, ciencia e innovación tecnológica a partir de la integración para formar redes de conocimientos, talento humano, infraestructura tecnológica, reutilización de activos de software y procesos flexibles que permitan alcanzar ventajas competitivas.

Al analizar lo expuesto en este sentido por Drucker (1994) los autores señalan que la industria de alta tecnología no sigue planteamientos de oferta y demanda de las economías clásica, neoclásica y keynesiana, en las cuales los costos de producción aumentan en proporción directa con el volumen de la producción, pues, en este tipo de organización, los costos de producción disminuyen en el tiempo, conforme al aumento del volumen de producción (González, 2006). Por su parte cómo lo denomina el ecologista Jaula Botet, el macabro "Mercado" propio de la economía del Capital, ejerce magistralmente la función de tirano del ambiente, por ser acreedor de decisiones sobre la explotación de los recursos naturales y de los seres humanos como simples mercancías, al atribuirse el derecho de determinar la calidad y la cantidad de productos y de servicios a satisfacer, en dependencia de las demandas, mayoritariamente emitidas por las sociedades consumistas.

En este sentido Drucker, promueve la idea de que esto requiere proteger la industria y dirigir el comercio con otros bloques, sobre la base de reciprocidad, en vez de proteccionismo o libre comercio, arguyendo que con ello el regionalismo sea inevitable e irreversible. Una de las soluciones al respecto tratan con la aparición de una nueva fuerza regional (ALBA, CELAC) capaz de movilizar a las mayorías oprimidas del subcontinente, en la búsqueda de una nueva sociedad, con una nueva economía dotada de una visión más social, dando al traste con un nuevo saber global incluyente de la diversidad del 
conocimiento universal yendo de la economía discriminatoria y competitiva, a la economía complementaria y solidaria.

Es preciso promover la innovación tecnológica y organizacional en las empresas, facilitar la entrada de nuevas empresas innovadoras y suscitar nuevos sectores de alta innovación. Se necesita establecer adecuadamente la división del trabajo innovador entre el Estado, sector privado y academia.

\section{CONCLUSIONES}

El progreso tecnológico, según las teorías del crecimiento de las economías, se basa en el conocimiento como factor productivo que depende del desarrollo endógeno que sean capaces de lograr y explica que la acumulación de capital en los países más industrializados del mundo ha incrementado la brecha existente en cuanto al crecimiento económico.

La universidad ecuatoriana como entidad generadora de conocimientos debe articularse en torno a la investigación, el desarrollo y la innovación, potenciar las redes existentes con el sector empresarial y propiciar la creación de otras que trabajen real y eficazmente en la creación e introducción de nuevos conocimientos aportando directamente al desarrollo social y económico del país.

Las TIC y los flujos de información, comunicación y conocimiento son la base de un proceso de transformación económica radical como alternativa viable para que el país dirija sus esfuerzos a la economía basada en conocimiento por la alta capacidad científica existente que demanda la formación de la base legal, organizativa y económica para la creación de organizaciones de alta tecnología.

\section{REFERENCIAS BIBLIOGRÁFICAS}

Arocena, R., \& Sutz J., (2003). Sistemas de innovación en ciencias de la vida: hacia un estudio desde los enfoques constructivos. Revista Iberoamericana de Ciencia, Tecnología, Sociedad e Innovación, No. 6, ISSN 1681-5645.

Brahima Sanou (2014). Measuring the Information Society Report 2014. International Telecommunication Union, Place des Nations, CH-1211 Geneva Switzerland, pp. 270. ISBN 978-92-61-15291-8
Castro Diaz-Balart, F, \& Pérez Rojas, H. (2006). Globalización, ciencia y desarrollo. Comprender el desafío a partir de una experiencia latinoamericana. In A. Gallina, J. Núñez, V. Capecchi \& L. F. Montalvo (Eds.), Innovaciones creativas y desarrollo humano (pp. 19-45). Ediciones Trilce, Montevideo.

Drucker, P. (1994). La Sociedad Postcapitalista. Crupo Editorial NORMA (4th ed.), Colombia, pp. 165-166 y 200-201.

David Ricardo, (2003). Principios de Economía Política y Tributación. Ediciones Pirámide, Crupo Anaya.

González Arencibia, M. (2006). Una gráfica de la Teoría del Desarrollo: Del crecimiento al desarrollo humano sostenible. Texto completo en ww.eumed.net/libros/2006/mga-des/

Guaipatin, C., \& Schwartz, L. (2014). Ecuador: Análisis del Sistema Nacional de Innovación: Hacia la consolidación de una cultura innovadora. Banco Interamericano de Desarrollo División de Competitividad e Innovación, pp.190.

Keynes, J. M. (1936). The General Theory of Employment, Interest and Money (1st ed.). Macmillan Cambridge University Press, for Royal Economic Society, pp. 263.

Lage Dávila, A. (2007). Propiedad y expropiación en la economía del conocimiento. Recuperado el 3 de mayo de 2015 de http:// www.cubadebate.cu/opinion/2007/01/04/ propiedad-y-expropiacion-en-la-economiadel-conocimiento/ \#.VUZQw_l_Oko.

León Guzmán, M. (2014). Economía del conocimiento y sistema nacional de innovación. Reflexión del 5 de mayo de 2014, El Telégrafo, http://www.telegrafo.com.ec/ economia/masqmenos/item/economia-delconocimiento.html

Lucas, R. E. (1988). On the Mechanics of Economic Development. Journal of Monetary Economics, 22, 3-42.

Lundvall, B-̊. (1992). National Systems of Innovation: Towards a Theory of Innovation and Interactive Learning. London: Pinter Publishers.

Lundvall, B. A. (2000). Los Sistemas Nacionales de Innovación: relaciones y aprendizaje, en: Los Sistemas de Ciencia e Innovación Tecnológica. Editado por el Ministerio de la Industria Básica (Cuba). La Habana. 